Somnologie $2013 \cdot 17: 245-245$

DOI 10.1007/s11818-013-0638-5

Online publiziert: 5. Dezember 2013

(c) Springer-Verlag Berlin Heidelberg 2013

\section{S. Happe}

Abt. für Neurologie, Klinik Maria Frieden, Telgte

\section{Editorial}

Das Restless-legs-Syndrom (RLS) ist mit einer Prävalenz von 5 bis $10 \%[2,4]$ eine der häufigsten neurologischen Erkrankungen. Die Diagnose wird klinisch gestellt. Erhebungen konnten zeigen, dass etwa 1,5\% der Bevölkerung einen Behandlungswunsch hinsichtlich der RLS-Symptome haben $[1,3]$. Die pathophysiologischen Grundlagen des RLS sind noch immer ungeklärt. Die Therapie erfolgt überwiegend medikamentös mit dopaminergen Medikamenten. Wegen der klinischen Relevanz und der auch in den vergangenen Jahren ungebrochen aktiven RLSForschung wurde dieses Sonderheft zum RLS geplant.

Dieses Heft umfasst sechs Beiträge zum RLS, drei Übersichtsarbeiten und drei Originalarbeiten. Zunächst beleuchtet S. Fulda die Gründe der Geschlechtsunterschiede in der Prävalenz des RLS, die bei Frauen etwa doppelt so hoch ist wie bei Männern. Eine aktuelle Übersicht zur medikamentösen Behandlung des RLS mit neueren therapeutischen Möglichkeiten gibt K. Stiasny-Kolster. H. DankerHopfe et al. sind an einer großen Stichprobe von RLS-Patienten aus Deutschland und der Schweiz der Frage nachgegangen, ob die Art der Anästhesie sowie Veränderungen der Medikation einen Einfluss auf den Schweregrad der postoperativen RLS-Symptomatik haben. Den Einfluss der Therapie mit Pramipexol auf periodische Beinbewegungen bei RLS und bei der „periodic limb movement disorder" (PLMD) untersuchte eine Arbeitsgruppe um C. Thiedemann. T.C. Wetter et al. beschreiben den aktuellen Wis- sensstand über SPECT-, PET- und MRTUntersuchungen zu Dopamin und Eisen beim RLS. Zuletzt stellen J. Steinig et al. neue Daten zu Persönlichkeitsmerkmalen, Depressionen und Angststörungen bei RLS-Patienten vor.

\section{Korrespondenzadresse

Prof. Dr. S. Happe
Abt. für Neurologie, Klinik
Maria Frieden
Am Krankenhaus 1,
48291 Telgte
shappe@gwdg.de

\section{Einhaltung ethischer Richtlinien}

Interessenkonflikt. Die Autorin gibt an, dass kein Interessenkonflikt besteht.

Dieser Beitrag beinhaltet keine Studien an Menschen oder Tieren.

\section{Literatur}

1. Allen RP, Bharmal M, Calloway M (2011) Prevalence and disease burden of primary restless legs syndrome: Results of a general population survey in the United States. Mov Disord 26:114-120

2. American Academy of Sleep Medicine (2005) ICSD-2 - International classification of sleep disorders, 2nd ed. Diagnostic and coding manual. American Academy of Sleep Medicine, Westchester IL

3. Happe S, Vennemann M, Evers S, Berger K (2008) Desired drug therapy of individuals with diagnosed and undiagnosed restless legs syndrome in a population-based survey. J Neurol 255:1365-1371

4. Montplaisier J, Allen RP, Walters A, Ferini-Strambi L (2011) Restless legs syndrome and periodic limb movements during sleep. In: Kryger MH, Roth T, Dement WC (eds) Principles and practice of sleep medicine, 5th ed. Elsevier Sauders, St. Louis, $p$ 1027-1037 